\title{
Shiitake Mushroom: A Tool of Medicine
}

\author{
Taufiqur Rahman ${ }^{1}$, MBK Choudhury ${ }^{2}$ \\ 1 National Mushroom Development Project, Savar, Dhaka \\ 2Directorate General of Health Services, Dhaka
}

\begin{abstract}
Medicinal mushrooms have an established history of use in traditional oriental therapies. Contemporary research has validated and documented much of the ancient knowledge. Over the last three decades, the interdisciplinary fields of science that study medicinal mushrooms has sprung up and has increasingly demonstrated the potent and unique properties of compounds extracted from a range of species. Currently, the field is being developed into a very fruitful area. Modern clinical practice in Japan, China, Korea and other Asian countries rely on mushroom-derived preparations.

Mushrooms have been studied for nutritional and medical purposes for its various potential anti-tumoral and immunomodulatory componests like polysaccharides that have been identified. For medical purposes, mushrooms have been consumed to prevent cancer and cardiac diseases, to improve blood circulation and to reduce blood cholesterol level. Some of these mushrooms have also been used for the treatment of physical and emotional stress, osteoporosis, gastric ulcers and chronic hepatitis, for the improvement of the quality of life of patients with diabetes and especially for the stimulation of immunity.

Shiitake has a history of medicinal uses. The mushroom is used as anticarcinogenic, antiinflammatory, antioxidant, antifungal, antibacterial, antiviral as well as antithrombotic in cardiovascular disorders. This article has been written to throw some light on Shiitake mushroom which has many nutritional values. Many Shiitake preparations came in market containing the active ingredients which can replace many other marketed synthetic medicines and may prove to have promising results with fewer side effects.
\end{abstract}

Key words: Lentinus edodes, lentinan, beta-glucan, eritadenine

\section{Introduction}

Shiitake mushroom, the common Japanese name for Lentinus edodes, is derived from the mushroom associated with the shii tree (Castanopsis cuspidate Schottky). Because Japan is the world leader in production of this type of mushroom, the mushroom is now widely known by this name. These mushrooms are renowned in Far East countries (e.g. Japan, China and Korea) as a food and medicine for thousands of years. In the year 199 A.D., Kyusuyu, a native tribe of Japan, offered the Japanese Emperor Chuai a shiitake mushroom. Even older documents record its use in ancient China where it was referred to as ''ko-ko"' or ''hoang-mo"'1.
The cultivation of this mushroom has been practiced for a thousand years with its cultivation originating in China during the Sung Dynasty (960-1127). Both history and legend credit $\mathrm{Wu}$ San Kwung as the originator of shiitake cultivation. Almost every mushroomgrowing village in China has a temple in his honor $^{2}$. In 1313, Chinese author Wang Cheng recorded shiitake-growing techniques in his Book of Agriculture. He described how to select a suitable site, choose appropriate tools and cut down the trees on which one could cultivate the mushrooms. He outlined the basic methods as follows: Cut the bark with a hatchet and cover 
the logs with soil. After 1year, top the soil and water frequently, beat the logs with a wooden club to induce mushroom production. The mushrooms will appear after a rain ${ }^{2,3}$. Shiitake mushroom cultivation techniques were probably introduced to Japanese farmers by the Chinese between 1500 and 1600 A.D ${ }^{4}$. At present, shiitake is one of the five most cultivated edible mushrooms in the world ${ }^{5}$. Its production (2 million tons) is second only to button mushroom Agaricus bisporus, grown mainly in East Asia. shiitake is now arousing interest worldwide $5,6,7,8$. Increasing markets have been spawned, partly by the exotic and well appreciated taste of shiitake and partly by advances in research that has demonstrated its significant medicinal properties. Shiitake mushroom is becoming popular in nutritional and medicinal products throughout Asia, Europe and North America.

\section{Discussion}

Shiitake are traditionally well-known edible mushrooms of high nutritional value. Raw or dried forms used in Chinese curative powers of shiitake mushroom are legendary. It was stated in Ri Youg Ben Cao, Vol. 3 (1620), written by Wu-Rui of the Ming Dynasty, 'shiitake accelerates vital energy, wards off hunger, cures colds and defeats body fluid energy." In later years, it was found that the mushroom contained various important nutrients. Moreover, recent scientific investigations have isolated many compounds and have found evidence of their health promotion activities $1,7,8,9,10$.

Shiitake mushrooms have excellent nutritional value. Their raw fruit bodies contain water, protein, lipids, carbohydrates as well as vitamins and minerals. It should be noted that amounts of nutrients and biologically active compounds differ in various strains and are affected by substrate, fruiting conditions and methods of cultivation. On a dry weight basis, they have a relatively high nutritional value when compared to commonly consumed vegetables.

Dried shiitake mushrooms are rich in carbohydrates and protein. They contain 58-60\% carbohydrates, $20-23 \%$ protein (digestibility of which is $80-87 \%$ ), 9-10\% fiber, 3-4\% lipids and $4-5 \%$ ash. The mushroom is a good source of vitamins, especially pro-vitamin $\mathrm{D}_{2}$ (ergosterol) $325 \mathrm{mg} \%$ which under ultraviolet (UV) light and heat yields calcitriol. It also contains $\mathrm{B}$ vitamins, including $\mathrm{B}_{1}, \mathrm{~B}_{2}, \mathrm{~B}_{12}$ and pantothenic acid ${ }^{1,3,9,10}$. Minerals found include $\mathrm{Fe}, \mathrm{Mn}, \mathrm{K}, \mathrm{Ca}, \mathrm{Mg}, \mathrm{Cd}, \mathrm{Cu}, \mathrm{P}$, and $\mathrm{Zn}$. Analysis of dried cultured shiitake mycelium gives the following mineral concentrations (in $\mathrm{mg} / \mathrm{g}$ of dry weight): $\mathrm{K}, 15.1 ; \mathrm{Ca}, 22 ; \mathrm{Mg}, 44-78$; $\mathrm{Mn}, 1.2 ; \mathrm{Cd}, 0.96 ; \mathrm{Fe}, 2.36 ; \mathrm{Ni}, 52.5 ; \mathrm{Cu}, 89.1$; P, 281; Zn, 282; Ge, 3; Br, 11.4 and Sr, 164. Water-soluble polysaccharides amount to $1-5 \%$ of the dry weight of the shiitake mushroom. In addition to glycogen-like polysaccharides, $(1-4) \rightarrow(1-6)-\alpha-D-$ glucans and antitumor polysaccharides, lentinan, $(1-3) \rightarrow(1-6)-\beta$-bonded heteroglucans, heterogalactans, heteromannans, xyloglucans, etc. have been identified. The mushrooms' indigestible polysaccharides which serve as dietary fiber include heteroglycan, polyuronide, $\beta$-glucan as well as chitin. Among the free sugars present are trehalose, glycerol, mannitol, arabitol, mannose, and arabinose $1,7,8,9,10$.

In shiitake mushrooms, dietary fiber consists of water-soluble materials such as $\beta$-glucan and protein and water-insoluble substances extractable only with salts, acids, and alkalies such as polyuronide (acidic polysaccharide), hemicellulose, $\beta$-glucan with heterosaccharide chains, lignin and chitin present as cell wall constituents. The fatty acids account for $3.38 \%$ of the total lipids 10,11 . Their composition is as follows: linoleic acid (18:2), 72.8\%; palmitic acid (16: 0$), 14.7 \%$; oleic acid (18:1), $3.0 \%$; tetradecenoic acid (14:1), $1.6 \%$; stearic acid (18:0), 0.9\%; and myristic acid (14:0), $0.1 \%$.

The aroma components include alcohols, ketones, sulfides, alkanes, fatty acids etc. The major volatile flavor contributors are matsutakeol (octen-1-ol-3) and ethyl-n-amyl ketone. The characteristic aroma of shiitake mushrooms was identified as 1,2,3,5,6pentathiepane. According to Mizuno10, the components responsible for the delicious flavor 
are monosodium glutamate, free amino acids, low molecular weight peptides, organic acids, and sugars. Their relative ratios are responsible for the variation in flavor naturally seen in this mushroom. Organic acids contributing to the flavor of shiitake mushroom include malic acid fumaric acid $\alpha$-keto-glutaric acid, oxalic acid, lactic acid, acetic acid, formic acid and glycolic acid.

\section{Anticarcinogenic and Antitumor Effects}

Using methods of fractionation and purification of polysaccharides, Chihara et al. ${ }^{12,13,14}$ isolated a watersoluble antitumor polysaccharide from fruiting bodies of shiitake which was named 'lentinan" after the genus Lentinus to which the shiitake mushroom belongs. Chihara was one of the first to report on the antitumor properties of the mushroom stating that lentinan "was found to almost completely regress the solid type tumors of Sarcoma 180 and several kinds of tumors including methylchloranthrene-induced fibrosarcoma in synergic host-tumor system $\mathrm{A}^{13,14{ }^{\prime \prime}}$. The antitumor effect of lentinan was originally confirmed by using Sarcoma 180 transplanted in CD-1/ICD mice12. Later it showed prominent antitumor activity not only against allogenic tumors such as Sarcoma 180 but also against various synergic and autochthonous tumors and it prevented chemical and viral oncogenesis 15 . The molecular formula of $\beta$-D-glucan lentinan is $\left(\mathrm{C}_{6} \mathrm{H}_{10} \mathrm{O}_{5}\right)_{\mathrm{n}}$ and the mean molecular weight is about one million. Its structure was confirmed as $\beta \rightarrow(1-3)$-D-glucopyranan with a branched chain of $\beta \rightarrow(1-6)$-monoglycosyl (branching degree: $2.5)$, a right-handed triple helix ${ }^{7,10,15,16}$. It is water soluble, heat stable and alkali labile. That is, $\beta$-D-glucan binds to lymphocyte surfaces or serum-specific proteins which activate macrophage, T-helper cells, natural killer (NK) cells and other effectors cells. All these increase the production of antibodies as well as interleukins (IL-1, IL-2) and interferon (IFN-g) released upon activation of effectors cells 17,18 . Thus, the carcinostatic effect of lentinan results from the activation of the host's immune system. In animal testing of carcinostatic activity, intraperitoneal administration is used but oral administration is occasionally effective.

The purified polysaccharide has been shown in animal studies to produce strong tumor regression and even the disappearance of sarcoma tumors in 5 weeks, ascitc hepatoma $134^{16,17,19}$ and Ehrlich carcinoma as well as a number of other experimentally induced cancers in allogenic, syngeneic and autologous hosts. It also exhibits preventive activity against chemical carcinogenesis. Injections of lentinan into mice produced either an $80 \%$ reduction in tumor size or complete regression in most of the animals tested. Howeover, an intact immune system and a functioning thymus gland were found to be requisite for its anticancer effect 20,21 . When immunosuppressive agents such as bbenzylthioguanosine or X-radiation were given with lentinan, the antitumor effect decreased. The polysaccharide has also been found to restore the enzyme activity of X-prolyldipeptidyl-aminopeptidase which can be depressed in cancer patients and in mice with implanted tumors 22 . Laboratory tests seem to indicate a role for the adrenal-pituitary axis and central peripheral nervous system including serotonin, 5HT, histamine and catecholamies in lentinan's antitumor activity $1,9,15,18$.

The oral administration of the polysaccharide to AKR mice exerted strong antitumor activity resulting in raised levels of lymphocytokines, such as IFN-g, tumor necrosis factor (TNF- $\alpha$ ) and IL-1 $\alpha$. Tissue cultures of murine macrophages CRL-2019, $\beta$-lymphocytes HB-284 and T-lymphocytes DRL-8179 which were treated with lentinan, showed high levels of activation using flow cytometry. Lentinan-activated immunocytes particularly the T-helper cells, might render the physiological constitutions of the host highly cancer and infection resistant. Adoptive immunotherapy of the immunodeficient mice such as the nude (athymic) mice, $\beta$-cell deficient mice and severe combined immunodeficient (SCID) mice via the transfer of the lentinanactivated immunocytes resulted in the inhibition of tumor growth. Lentinan appeared to represent 
a unique class of host defense potentiators (HDP), protecting the hosts from the side effects of conventional therapeutic measures and improving various kinds of immunological parameters with no toxic side effects in animal models ${ }^{17,18,23,24}$.

\section{Hepatoprotective Effects}

The injection of lentinan slowed the growth of cancerous liver tumor in rats $11,16,25$. A polysaccharide fraction from shiitake mushrooms demonstrated liver protection in animals as well as the ability to improve liver function and enhance the production of antibodies to hepatitis $\mathrm{B}^{7,26}$. Lentinan improved serum glutamic pyruvic transaminase (SGPT) and completely restored GPT levels in mice with toxic hepatitis. Crude extracts of shiitake mushroom cultures have demonstrated liverprotecting actions. $9,11,16,26$

\section{Cardiovascular Effects}

The major cause of death in western countries is coronary artery disease, a primary risk factor for which is hypercholesterolemia that contributes to hardening of the arteries. In humans, $50 \%$ or more of the total serum cholesterol is derived from de novo synthesis $16,26,27$. It is known that shiitake mushroom is able to lower serum cholesterol via a factor known as eritadenine (also called ''lentinacin"' or ''lentysine''). Apparently, eritadenine reduces serum cholesterol in mice, not by the inhibition of cholesterol biosynthesis but by the acceleration of the excretion of ingested cholesterol and its metabolic decomposition. It has been shown to lower blood levels of cholesterol and lipids in animals. When added to the diet of rats, eritadenine $(0.005 \%)$ caused a $25 \%$ decrease in total serum cholesterol in as little as one week. The cholesterol-lowering activity of this substance is more pronounced in rats fed a high-fat diet than in those on a low-fat diet. Although feeding studies with humans have indicated a similar effect further research is needed. Hobbs 1,9 and Yang et al. ${ }^{27}$ have shown that shiitake mushrooms lowered serum cholesterol levels. Various studies have confirmed ${ }^{1}, 7,9,11$. that the mushroom can lower blood pressure and free cholesterol in plasma as well as accelerate the accumulation of lipids in the liver by removing them from circulation.

\section{Immune-Modulating Effects}

As was stated earlier, lentinan and other polysaccharides from shiitake mushrooms do not attack cancer cells directly, but produce their antitumor effects by activating different immune responses in the host. Lentinan, for example, appears to act as an HDP, which is able to restore or augment the responsiveness of host cells to lymphocytokines, hormones and other biologically active substances by stimulating maturation differentiation or proliferation of cells involved in host defense mechanisms ${ }^{17,18}$. Host defense potentiators are functionally different from biological response modifiers. Thus, lentinan is able to increase host resistance against various kinds of cancer and infectious diseases including acquired immuno deficiency syndrome (AIDS) ${ }^{7,24}$. The initial interactions of lentinan in the human body or animals are not presently known. However, there is a transitory but notable increase in several serum protein components in the $\alpha$ - and $\beta$-globulin region, namely, complement $\mathrm{C}_{3}$, hemopexin, and ceruloplasmin $7,9,17,18$.

Lentinan can activate NK cells in vitro in the same concentrations that are achieved in the blood plasma of patients treated clinically with lentinan. ${ }^{11,18}$ Natural killer-cell activity is involved in tumor suppression and while these cells do not stimulate T-killer cell activity or do so only under certain conditions, they are strong T-helper cell stimulants both in vitro and in vivo $1,7,9,11,17,18$. Using the blood of healthy donors and cancer patients, some authors have shown that the polysaccharide is able to stimulate peripheral blood lymphocytes in vitro to increase IL-2 mediated lymphokine-activated killer cell (LAK-cell) and NK cell activity at levels achievable in vivo by administration of clinical doses of lentinan. It has been shown to 
inhibit suppressor $\mathrm{T}$ cell activity in vivo and to increase the ratio of activated $\mathrm{T}$ cells and cytotoxic T-cells in the spleen when administered to gastric cancer patients undergoing chemotherapy $7,9,18$.

Many interesting biological activities of lentinan have been reported including: a) an increase in the activation of nonspecific inflammatory responses such as acute phase protein (APP) production, b) vascular dilation and hemorrhage in vivo, c) activation and generation of helper and cytotoxic T-cells, b) augmentation of immune mediators like IL-1 and IL-3, colony stimulating factor(s) and migration inhibitory factor, and e) increasing the capacity of peripheral blood mononuclear (PBM) cells of patients with gastric cancer and producing IL$1 \alpha$, IL- $1 \beta$, and a TNF- $\alpha^{7,9,17,18,23}$.

In an in vivo study of rats with peritonitis, combined lentinan-gentamicin treatment had a significantly better survival rate than the controls. Lentinan activated the peritoneal macrophages' secretory activity of active oxygen and produced cytokines, thus enhancing the ability of polymorphonuclear leukocytes (PMNs) to produce active oxygen, which has a bactericidal effect ${ }^{28}$. It also increases peritoneal macrophage cytotoxicity against metastatic tumor cells in mice, but not against a highly metastatic tumor type ${ }^{29}$. Some patients treated with lentinan for carcinomatous pleuritis or carcinomatous peritonitis has improved with the disappearance of malignancy, while in another group their condition deteriorated or diminished ${ }^{30}$. The polysaccharide can activate the normal and alternative pathways of the complement system and can split C3 into C3a and $\mathrm{C} 3 \mathrm{~b}$ enhancing macrophage activation 31 . Many biological reactions are accelerated and induced by lentinan including the very important phenomenon of infiltration of eosinophils, neutrophils and granulocytes around target tissues.

Lentinan's immune-activating ability may be linked with its modulation of hormonal factors, which are known to play a role in tumor growth. Aoki 31 showed that the antitumor activity of lentinan is strongly reduced by administration of thyroxin or hydrocortisone. It can also restore tumor-specific antigen-directed delayed-type hypersensitivity reaction (DTHR). Lentinan is not formally included among the nonspecific immunostimulants (RES stimulants), but it augments the induction of antigen-specific cytotoxic T-lymphocytes, macrophages and other nonspecific immune responses.

\section{Antibacterial Property}

Lentinan is therapeutically effective against Mycobacterium tuberculosis and Listeria monocytogenes. ${ }^{32}$ Oxalic acid is an agent responsible for the antimicrobial effect of Lentinula edodes ${ }^{33}$ grown in submerged liquid culture was bacteriostatic against Streptococcus pyogenes, Staphylococcus aureus and Bacillus megaterium $^{34}$. Lentinan inhibits Candida albicans and S.aureus ${ }^{35}$. Lentinan helps to regulate the inflammatory response in the host when encounter pathogens. It also activates the complement system which split C3 into C3a and $\mathrm{C} 3 \mathrm{~b}$, enhancing macrophage activation. ${ }^{36}$

\section{Antiviral Property}

Lentinan is able to inhibit replication of Adenovirus type 12, Abelson virus and VSVencephalitis virus ${ }^{32}$. Although lentinan itself has no ability to block HIV infection, concomitant treatment with 3'-azido-3'- deoxythymidine suppresses the surface expression of HIV antigens more than does AZT alone. ${ }^{32}$ Lentinula edodes naturally contain antiviral agents known as proteinase inhibitors. Anti HIV activities were reported for mycelia culture medium of $\mathrm{L}$. edodes (LEM) and water soluble lignin in LEM $^{37,38}$. Sulfated lentinan from L. edodes completely prevented HIV induced cytopathic effect 38 . The polysaccharide Lentinan demonstrates effects against influenza virus and polio virus as well as against some bacteria and parasites. These effects are mediated by immune system induction that even delays AIDS 
symptomatology appearance. This action would be linked to induction of increased level of interferon. L. edodes seems to be one of the most promising stimulator of immunofunctions. This mushroom is tested on HIV positive patients in the USA and in Japan. ${ }^{39}$

\section{Antioxidant Property}

Oxidative damage caused by free radicals may be related to aging and diseases, such as atherosclerosis, diabetes, cancer and cirrhosis 40 . Antioxidant compounds reduce the action of reactive oxygen species (ROS) in damaged tissues during the recovery process ${ }^{41}$. Kitzberger et al used extracts of $L$. edodes obtained by organic solvents to test the antioxidant activity ${ }^{42}$. Cheung and Cheung ${ }^{43}$ also reported the antioxidant activity of $L$. edodes against lipid peroxidation. They found that the low molecular weight sub fraction of water extracts of $L$. edodes had the highest antioxidant activity against lipid peroxidation of rat brain homogenate, with IC50 values of $1.05 \mathrm{mg} / \mathrm{ml}^{19}$. Shiitake enhance the host's antioxidant capacity or upregulating phase- 1 and phase- 2 enzymes involved in the metabolic transformation and detoxification of mutagenic compounds. ${ }^{44} L$. edodes is also inducer of superoxide dismutase and glutathione peroxidase, the two antioxidant enzymes $^{38}$. Lentinula edodes filtrates and mycelia exhibit Aflatoxin inhibiting effects 45 . They act as an external stimulus affecting the antioxidant states in the toxin producing fungus and leads to inhibition of Aflatoxin ${ }^{46}$.

\section{Antifungal Property}

From the fruiting bodies of the shiitake mushroom, a novel protein designated lentin with potent antifungal activity was isolated in 2003. ${ }^{24}$ It was unadsorbed on DEAE-cellulose, and adsorbed on Affi-gel, blue gel and Mono S. The N-terminal sequence of the protein showed similarity to endoglucanase. Lentin with a molecular mass of $27.5 \mathrm{kDa}$, inhibited mycelia growth in a variety of fungal species including Physalosporia piricola, Botrytis cinerea and Mycosphaerella arachidicola ${ }^{24}$.

\section{Anti-thrombotic Effects}

Thrombosis, has been shown to be significantly reduced in individuals consuming Shiitake mushroom oil. ${ }^{45}$ The department of Agricultural and Biological Chemistry at Nihon University in Japan has demonstrated that the levels of Lenthionine found in Shiitake mushrooms inhibited platelet aggregation ${ }^{45}$.

\section{Antihypercholesterolemic Effects}

Scientific studies involving animals have proved that eritadenine found in shiitake mushrooms is beneficial in reducing the cholesterol levels in the blood vessels as well as low density lipoproteins or LDL cholesterols that are harmful for the body. These researches have confirmed that eritadenine in shiitake has the ability to reduce the cholesterol levels in the blood vessels by $25 \%$ in just one week ${ }^{26}$. And surprisingly, the results after the usage of eritadenine was found to be better in subjects that consumed high protein food compared to those who took low protein food stuff. According to researches by Japanese medical scientists, chemicals present in shitake help in building up the harmful low density lipoproteins in the liver, where they are modified into high density lipoproteins or HDL cholesterols that are useful for the human system.

\section{Antihypertensive Effects}

High blood pressure means your heart has to do extra work and can lead to, if unchecked, a stroke or heart attack. Preliminary studies have suggested that dietary shiitake and other types of mushroom consumption may prevent blood pressure increase ${ }^{9}$.

\section{Antiobesity Effects}

Shiitake mushrooms possess many health benefits that can aid weight loss. Eating a wellbalanced diet that contains these healthful mushrooms will be the best way to lose weight and keep it off, according to the American Heart Association $^{47}$. Remember to prepare the mushrooms using a low fat, heart-healthy cooking method on your way to weight loss success. 


\section{Improves Appearance of Skin}

Applying shiitake mushroom extract to your skin may improve its appearance, according to Skincare-News.com ${ }^{47}$. Beauty products aimed at brightening your skin may contain mushroom extract because of its concentration of kojic acid, a natural alternative to hydroquinone, a chemical that bleaches your skin to fade scars and age spots. The antioxidant effects of shiitake are not limited only to protect your internal organs but skin creams and lotions that contains mushroom extract as an ingredient may be able to minimize inflammation of the skin.

\section{Toxicity and Side Effects}

Shiitake mushroom is edible, but some individuals may experience minor side effects or allergic reactions. Literature describes $7,9,12,46$ cases of shiitake-induced toxicodermia and shiitake dermatitis. Allergic reactions to the spores of shiitake mushrooms have been reported in workers picking mushrooms indoors, who are prone to an immune reaction to spores called 'mushroom worker's lung". Symptoms include fever, headache, congestion, coughing, sneezing, nausea and general malaise ${ }^{48}$. A water extract of the fruiting body was found 49 to decrease the effectiveness of blood platelets in initiating coagulation. L. edodes mycelium has shown no evidence of being acutely toxic, even in massive doses of over $50 \mathrm{mg} /$ day for 1 week, though mild side effects such as diarrhoea and skin rash may occur. As a rule, symptoms disappear after a short period when the body has adapted to the extract. Lentinan has no known serious side effects. However, in clinical trials of patients with advanced cancer, minor side reactions occurred such as a slight increase in glutamate-oxaloacetate transminase (GOT) and GPT liver enzymes and a feeling of mild pressure on the chest. But these changes disappeared after lentinan administration was stopped. ${ }^{49}$

\section{Dosage and Preparation of Shittake Mushroom}

Shiitake mushroom is prescribed in various forms. It may be injected as a solution (1 $\mathrm{mg} / \mathrm{vial}$ ) or ingested as a sugar-coated tablet, capsule, concentrate, powdered extract, syrup, tea, wine, and/or as a medicinal dish. Lentinan's anticancer effect is highly dose dependent. The standard dose of the dried fruiting body in tea or in mushroom dishes is given as 6-16 gm, equivalent to about $90 \mathrm{gm}$ of fresh fruiting body. As a tablet, the dosage is usually in the form of 2 gm tablets 2-4/day. Commercial preparations can be found in many countries in health food stores and supermarkets. The tablets are usually made from a dried water extract of the mycelia or fruiting bodies because drying concentrates the lentinan and other active principles. Standardized extracts are also available and they are preferred because the amount of lentinan present is certified and clearly stated on the bottle. Although the fresh form can be a valuable dietary supplement, the quantities one would require for therapeutic doses are so great that its consumption could cause digestive upset. That is why LEM, which is concentrated and easily absorbed, is preferred for medicinal use. ${ }^{7,8,9}$ Fresh and dried shiitake mushrooms are used in medicinal mushroom dishes (Yakuzen). Certain medicinal effects have been recently studied 11 and found to reduce the ill effects of certain gourmet diets. These dishes can be prepared in many ways, limited only by one's ingenuity: boiled, grilled, skewered, or on aluminum foil with different types of seasoning. To obtain a concentrate, whole fruit bodies or powdered mushrooms are boiled in water. The extract is then concentrated and is used as a drink. It can also be consumed as a tea: canned "shiitake tea" which contains a concentrated extract or many other shiitake "'healthy tea" products sold as mushroom containing tea bags. Shiitake mushroom concentrate can be freezedried or spray-dried to form a granular powder. There are many products containing powdered shiitake mushroom extract, such as a mixture of this powder with vitamin $\mathrm{C}$ crystals or with medicinal plants such as ginseng. In Eastern countries, the mushroom is mainly used as a concentrate when extracted with boiling water. 
31 Bangladesh J Med Biochem; 5(1)

Residues from these processes still contain substantial amounts of useful polysaccharide substances, including those effective as antitumor compounds such as b-glucans, nucleic acids, dietary fiber etc. An alcohol extraction product is obtained by preserving fresh or dried shiitake mushroom in alcohol, which has been mixed with sugar or molasses. Some products, including ' healthy shiitake wine' ' are sold as a nightcap or as a tonic drink $^{8,10}$.

\section{References}

1. Hobbs C. Medicinal Mushrooms. An Exploration of Tradition, Healing, and Culture, 2nd Ed. Botanica Press, Inc.: Santa Cruz, CA, USA. 1995.

2. Miles PG and Chang ST. Mushroom Biology: Concise Basics and Current Development; World Scientific: Singapore 1997; p 193.

3. Przbylowicz P and Donoghue J. Shiitake Grower's Handbook: The Art and Science of Mushroom Cultivation; Hunt Publ. Co. Dubugue, Kendall. 1990; p 199.

4. Singer R and Harris B. Mushrooms and Truffles: Botany, Cultivation, and Utilization, 2nd Ed. Koeltz Sci. Books: Koenigstein. 1987.

5. Chang ST. World production of cultivated edible and medicinal mushrooms in 1997 with emphasis on Lentinus edodes (Berk.) Sing. In China. Int J Med Mushr 1999;1:387-409.

6. Royse D. Specialty mushrooms and their cultivation. Horticult Rev 1997; 19: 59-97.

7. Wasser SP and Weis AL. Medicinal Mushrooms. Lentinus edodes (Berk.) Singer; Nevo, E., Ed. Peledfus Publ. House: Haifa, Israel 1997; p 95.

8. Stamets P. Growing Gourmet and Medicinal Mushrooms, 3rd Ed. Ten Speed Press: CA, USA 2000.

9. Hobbs C. Medicinal value of Lentinus edodes (Berk.) Sing. A literature review. Int J Med Mushr 2000;2:87-302.

10. Mizuno T. Shiitake, Lentinus edodes: functional properties for medicinal and food purposes. Food Rev Int 1995;11:7-21.

11. Mizuno T. A development of antitumor polysaccharides from mushroom fungi. Food Ingred J Jpn 1996;167:69-85.

12. Chihara G, Maeda YY, Hamuro J, Sasaki T, Fukuoka F. Inhibition of mouse Sarcoma 180 by polysaccharides from Lentinus edodes (Berk.). Sing Nature 1969; 222: 687-688.
Taufiqur Rahman and MBK Choudhury

13. Chihara G, Hamuro J, Maeda YY, Arai Y, Fukuoka F. Antitumor polysaccharide derived chemically from natural glucan (pachyman). Nature 1970; 225: 943-944.

14. Chihara G, Hamuro J, Maeda YY, Arai Y, Fukuoka F. Fractionation and purification of the polysaccharides with marked antitumor activity, especially lentinan, from Lentinus edodes (Berk.) Sing. (an edible mushroom). Cancer Res 1970; 30: 2776-2781.

15. Wasser SP. Medicinal mushrooms as a source of antitumor and immunomodulating polysaccharides. Appl Microbiol Biotechnol 2000;60: 258-274.

16. Smith J, Rowan N, Sullivan R. Medicinal mushrooms. Their therapeutic properties and current medical usage with special emphasis on cancer treatment; Special Report Commissioned by Cancer research UK. The University of Strathclyde in Glasgow 2002; p 256.

17. Yap AT and Ng ML. Immunopotentiating properties of lentinan (1-3)-b-D-glucan extracted from culinary-medicinal shiitake mushroom Lentinus edodes (Berk.) Singer (Agaricomycetideae). Int J Med Mushr 2003;5:352-372.

18. Yap AT and Ng ML. An improved method for the isolation of lentinan from the edible and medicinal shiitake mushroom, Lentinus edodes (Berk.) Sing. (Agaricomycetideae). Int J Med Mushr 2001;3:9-20.

19. Moriyama M. Anti-tumor effect of polysaccharide lentinan on transplanted ascites hepatoma- 134 in $\mathrm{C} 3 \mathrm{H} / \mathrm{He}$ mice. In Manipulation of Host Defence Mechanisms, International Congress Series 1981; 576

20. Pegler D. The classification of the genus Lentinus Fr. (Basidiomycota). Kavaka 1975;3:11-20.

21. Earle FS. The genera of the North American gillfungi. Bull N Y Bot Gard 1999;5:373-451.

22. Mori $K$, Toyomasu $T$, Nanba $H$, Kuroda $H$. Antitumor activity of fruit bodies of edible mushrooms orally administrated to mice. Mushr J Trop 1987;7:121-126.

23. DeVere White RW, Hackman RM, Soares SE, Beckett LA, Sun B. Effect of a mushroom mycelium extract on the treatment of prostate cancer. Urology 2002;60:640-644.

24. Ngai PHK and Ng TB. Lentin, a novel and potent antifungal protein from shiitake mushroom with inhibitory effects on activity of human immunodeficiency virus-1 reverse transcriptase and proliferation of leukemia cells. Life Sci 2003;73:3363-3374. 
25. Amagase H. Treatment of hepatitis B patients with Lentinus edodes mycelium. Proceedings of the XII International Congress of Gastroenterology, Lisbon. 1987; p 197.

26. Wasser SP and Weis AL. Medicinal properties of substances occurring in higher Basidiomycetes mushrooms: current perspectives (review). Int J Med Mushr 1999;1:31-62.

27. Yang BK, Kim DH, Jeong SC, Das S, Choi YS, Shin JS, Lee SC, Song CH. Hypoglycemic effect of a Lentinus edodes exopolymer produced from a submerged mycelia culture. Biosci Biotechnol Biochem 2002;66:937-942.

28. Kurashige S, Akusawa Y, Endo F. Effects of Lentinus edodes, Grifola frondosa, and Pleurotus ostreatus administration on cancer outbreak, and activities of macrophages and lymphocytes in mice treated with a carcinogen, N-butylNbutanolnitrosoamine. Immunopharm Immunotoxicol 1997; 19:175-183.

29. Ladanyi A, Timar J, Lapis K. Effect of lentinan on macrophage cytotoxicity against metastatic tumour cells. J Cancer Immunol Immunother 1993;36:123126.

30. Yoshino S, Tabata $\mathrm{T}$, Hazama S, Iizuka N, Yamamoto K, Hirayama M, Tangoku A, Oka M. Immunoregulatory effects of the anitumour polysaccharide lentinan on $\mathrm{Th} 1=\mathrm{Th} 2$ balance in patients with digestive cancers. Anticancer Res 2000; 20: 4707-4711.

31. Aoki T. Lentinan. In Immune, Modulation Agents and Their Mechanisms; Fenichel, R.L., Chirgis, M.A., Eds.; Immunol Studies 1984; 25: 62-77.

32. Ooi CEV and Liu F. Immunomodulation and anticancer activity of polysaccharide-protein complexes. Current medicinal chemistry 2000; 7: 715-729.

33. Leefa F, Huijuan P, Andrea ST, Ashok P, Carlos SR. Advances in mushroom research in last decade. Food technol biotechnol 2006; 44: 303-311.

34. Nikitina EV, Tsivileva MO, Pankratov NA, Bychkov AN. Lentinula edodes biotechnology- from lentinan to lectins. Food technol biotechnol 2007; 45:230-237.

35. Ichimura $\mathrm{T}$, Watanabe $\mathrm{O}$, Maruyama S. Inhibition of HIV-1 protease by water-soluble lignin-like substance from an edible mushroom Fuscoporia oblique. Biosci Biotechnol Biochem 1998;62: 575577.
36. Wong KC. Immunomodulatory properties of the Chinese medicinal extract polysaccharide peptide. Hub HKU 2010.

37. Colins RA and Ng TB. Polysaccharopeptide from Coriolus versicolor has potential for use against human immunodeficiency virus type 1 infection. Life Sci 1997; 60: 383-387.

38. Patrick P, Francoise F, Sylvie R. Biological and pharmacological activity of higher fungi: 20-year retrospective analysis. Cryptogamie Mycologie 2006; 27: 1-23.

39. Halliwell B and Gutteridge JMC. Oxygen toxicity, oxygen radicals, transition metals and disease. Biochem J 1984; 219: 1-4.

40. Barros L, Baptista P and Ferreira. ICFR: Effect of Lactarius piperatus fruiting body maturity stage on antioxidant activity measured by several biochemical assays. Food Chem Toxicol 2007; 45: 1731-1737.

41. Sreenivasan S, Sugumaran A, Lachimanan Y, Latha R, Vijenthi D, Saravanan SA. In vitro antioxidant activity and hepatoprotective effects of Lentinula edodes against paracetamol-induced hepatotoxicity. Molecules 2010; 15: 4478-4489.

42. Cheung LM and Cheung PCK. Mushroom extracts with antioxidant activity against lipid oxidation. Food Chem 2005; 89: 403-409

43. Lee IS and Nishikawa A. Polyozellus multiplex, a Korean wild mushroom, as a potent chemopreventive agent against stomach cancer. Life Sci 2003; 73: 3225-3234.

44. Fanelli C, Fabbri AA, Finotti E, Fasella P, Passi S. Free radicals and aflatoxin biosynthesis. Cell Mol Life Sci 1984; 40:191-193.

45. The health benefits of shiitake mushrooms. Elements 4 health. 2011.

46. Ueda A. Allergic contact dermatitis in shiitake (Lentinus edodes) (Berk. Sing.) growers Contact Dermatitis. 1992; 26: 228-233.

47. http://www.livestrong.com/article/289297-shiitakemushrooms-weight-loss/\#ixzz25JROYBuG

48. Leon PC. Mushroom worker's lung. Detection of antibodies against Shii-take (Lentinus edodes) spore antigens in Shii-take workers. J Occup Med 1992; 34:1097-1101.

49. Yang QY and Jong SC. Medicinal mushrooms in China. Mushr Sci XII 1989; 631-642. 